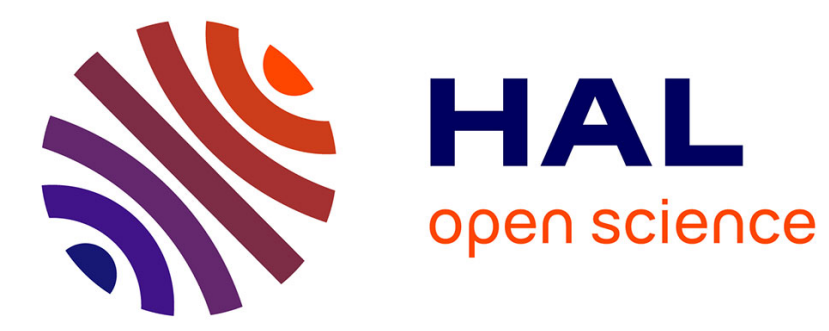

\title{
Topology-based Abstraction of Complex Biologiical Systems: Application to the Golgi Apparatus
}

Mathieu Poudret, Agnès Arnould, Jean-Paul Comet, Pascale Le Gall, Philippe Meseure, François Képès

\section{- To cite this version:}

Mathieu Poudret, Agnès Arnould, Jean-Paul Comet, Pascale Le Gall, Philippe Meseure, et al.. Topology-based Abstraction of Complex Biologiical Systems: Application to the Golgi Apparatus. Theorie in den Biowissenschaften / Theory in Biosciences, 2008, 127 (2), pp.79-88. hal-00352003

\section{HAL Id: hal-00352003 https://hal.science/hal-00352003}

Submitted on 12 Jan 2009

HAL is a multi-disciplinary open access archive for the deposit and dissemination of scientific research documents, whether they are published or not. The documents may come from teaching and research institutions in France or abroad, or from public or private research centers.
L'archive ouverte pluridisciplinaire HAL, est destinée au dépôt et à la diffusion de documents scientifiques de niveau recherche, publiés ou non, émanant des établissements d'enseignement et de recherche français ou étrangers, des laboratoires publics ou privés.

$$
\text { Copyright }
$$




\title{
Topology-based Abstraction of Complex Biological Systems: Application to the Golgi Apparatus
}

\author{
Mathieu Poudret • Agnès Arnould • \\ Jean-Paul Comet • Pascale Le Gall . \\ Philippe Meseure • François Képès
}

Received: date / Accepted: date

\begin{abstract}
Many complex cellular processes involve major changes in topology and geometry. We have developed a method using topology-based geometric modelling in which the edge labels of an $n$-dimensional generalized map (a sub-class of graphs) represent the relations between neighbouring biological compartments. We illustrate our method using two topological models of the Golgi apparatus. These models can be animated using transformation rules which depend on geometric and/or biochemical data and which modify both these data and the topology. Both models constitute plausible topological representations of the Golgi apparatus, but only the model based on a recent hypothesis about the Golgi apparatus is fully compatible with data from electron microscopy. Finally, we outline how our method may help biologists choose between different hypotheses.
\end{abstract}

Keywords Topology-based geometric modelling · Biological processes modelling

\section{Introduction}

Systems biology aims at understanding biological phenomena at different scales: intracellular environments, cells, organs, multi-cellular individuals and populations. Using computational tools to model and simulate such systems is therefore essential. This entails recognition of the principal characteristics of the phenomenon, choice of the appropriate level of abstraction, and comparison of different models. Compartmentalization is important in modelling biological systems and a spatial representation of the compartments is often needed to describe both their static and dynamic characteristics

J.-P. Comet

I3S UMR 6070 CNRS, Université de Nice-Sophia-Antipolis, 06903 Sophia-Antipolis, France E-mail: comet@unice.fr

M. Poudret · F. Képès · P. Le Gall

Programme d'Épigénomique, Génopole, 91000 Évry, France

E-mail: \{francois.kepes,pascale.legall\}@epigenomique.genopole.fr

A. Arnould · P. Meseure

SIC, Université de Poitiers, 86962 Futuroscope, France

E-mail: \{arnould,meseure\}@sic.univ-poitiers.fr 
[Presley et al., 2002]. In particular, the dynamics of the relations between neighbouring compartments influence the evolution of system parameters (such as the concentration of molecules).

A variety of approaches have been used to model cellular systems: differential models have been used to study the evolution of concentrations (see Virtual Cell [Eungdamrong and Iyengar, 2007]), Boolean or discrete modelling for genetic regulatory networks, and rule-based modelling for biochemical reactions (for which transformational rules are particularly suited). In the case of rule-based modelling, formal methods like model checking [Bernot et al., 2004] or symbolic execution [Mateus et al., 2007] have been fruitfully applied to verify that the model satisfies a known property of the biological system. However, many rule-based models ignore compartmentalization and treat the system, unrealistically, as a homogeneous environment. Recent rule-based modelling takes into account different compartments (see Brane calculi [Cardelli, 2005], Bioambients [Regev et al., 2004] and BioCham [Calzone et al., 2006]). In these models, the compartmentalization only captures static topology or simple topological modifications (resulting, for example, from endocytosis or exocytosis) but not geometric aspects (such as the position and shape of the objects).

Topology-based geometric modelling [Lienhardt, 1989] is particularly adapted to represent compartmentalization and is widely advocated for computer graphics. It deals with the representation of the structure of objects (their decomposition into topological units: vertices, edges, faces and volumes) and of the neighbouring relations that exist between topological units. It treats topological structure and geometry separately, and this means that the topological properties of objects can be studied without knowledge of their geometry (this does not exclude a subsequent study of complex geometric shapes). Moreover, in topology-based geometric modelling, both objects and the frontiers between objects can be manipulated in the same way (this facilitates the modelling of the transport of molecules between biological compartments). Our long term goal is to investigate those topology-based models of interest to biologists who possess observation-based knowledge. In previous work [Poudret et al., 2007], we formally expressed basic topological operations in terms of generic rules that can be applied to a large family of topological objects and we illustrated this topology-based approach using a simple interaction between two cells. In this paper, we evaluate this approach in a case in which a more complex spatial representation is required.

The Golgi apparatus is the place in the cell where proteins remain during their maturation phase before their excretion. It is widely accepted that excretion of proteins is strongly linked to the spatial dynamics of the Golgi apparatus. However, the topology of the apparatus is not fully understood. Three classes of hypotheses have been proposed [Képès et al., 2004]. In pursuing the goal of evaluating our topologybased approach, we first built two models to represent the Golgi apparatus based on different topological principles. In one model, the apparatus consists of a stack of disconnected saccules surrounded by vesicles while in the other model the saccules are connected by tubular structures and there are no vesicles. Analysis of 2-dimensional sections show that only the second model (which implements a more recent hypothesis about the Golgi apparatus) is fully compatible with the available biological observations. As both models are static, we add certain transformation rules to allow them to deal with dynamics and to incorporate the essence of the Golgi apparatus hypotheses.

The Golgi apparatus and the current hypotheses on its topology, our rule-based topological approach and a topology-based implementation of the Golgi apparatus hypotheses are presented in Sections 1, 2 and 3, respectively. Finally, in Section 4, we 
briefly present our computer-aided methodology for helping biologists to understand and choose hypotheses about the topology and dynamics of the Golgi apparatus.

\section{The Golgi Apparatus}

\subsection{General description}

Discovered by Camillo Golgi in 1898, the Golgi apparatus (or dictyosome in plants) is an organelle whose role includes the transport of proteins synthesised by the cell from the endoplasmic reticulum to the plasma membrane or to lysosomes. Not only the Golgi apparatus sorts the proteins in order to transport them into adapted locations, but it is also the place of protein maturation by the means of loss of peptidic sequences and addition of sugars (glycosylation) or sulfate (sulfatation). The Golgi apparatus is located near the nucleus and the centrosome. By electron microscopy (see Fig. 1), it generally appears as a stack of 5 or 6 disconnected cisternaes (the saccules) bounded with a phospholipidic membrane (see $\mathbf{S}$ on Fig. 1(a)). This stack is usually surrounded by small vesicles that bud out from the saccules (see V on Fig. 1(a)). Notice that on some pictures, the saccules appear perforated (see $\mathbf{P}$ on Fig. 1(b)). At last, the Golgi apparatus is a polarised object: the cis face is directed to the endoplasmic reticulum while the opposite trans face is often directed to the plasma membrane.

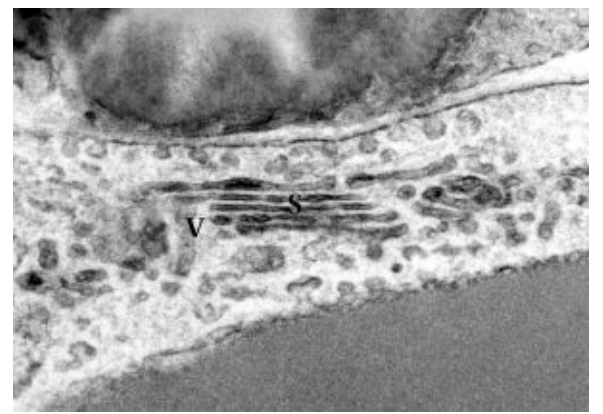

(a)

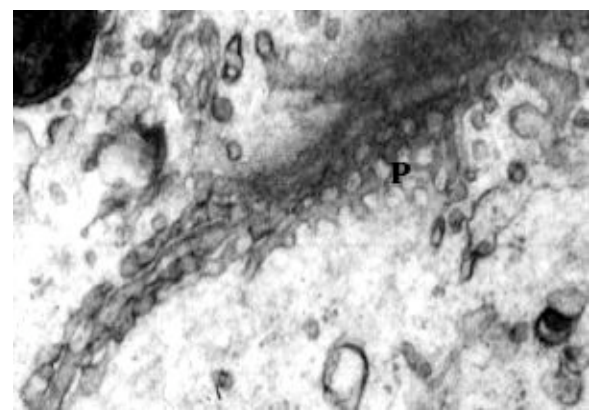

(b)

Fig. 1 Electron micrograph showing a cross-section of the Golgi apparatus in mouse endothelial cell 1(a), courtesy of Jean-Marc Verbavatz (IBITEC-S Institute, CEA-Saclay) and an oblique view of the Golgi apparatus in a rat Sertoli cell 1(b), from [Rambourg et al., 1979]

\subsection{Three hypotheses on the Golgi apparatus}

Because of observation limitations, the complete structure of the Golgi apparatus is not precisely known. Indeed, with optical microscopy techniques, biologists observe the dynamics at the cost of a small resolution that does not allow them to observe the structure. By contrast, electron microscopy provides high resolution pictures but the observation is done on thin and inert section of the Golgi apparatus. Last but not least, those thin sections lead to many interpretation mistakes when a 3-dimensional 
reconstruction is performed (for instance, both spheres and tubes section can appear as discs on a picture).

In particular, the path that proteins follow from the endoplasmic reticulum to the plasmic membrane or lysosomes is not well known. Consequently, three main hypotheses exist [Képès et al., 2004]. The two first hypotheses appear quite similar since they both suppose that vesicles play a major role in the excretion of proteins. In the vesicular secretion hypothesis (see Fig. 2(a)), an aggregate of endoplasmic reticulum (ER) fragments generates disconnected saccules $(\mathbf{S})$. Proteins migrate through the stack by means of vesicles $(\mathbf{V})$ that jump from one saccule to another. They are finally evacuated by the means of secretory granules $(\mathbf{G})$ that bud out from the trans face. We know that enzymes in charge of the activation and the maturation of proteins are located near the cis face of the Golgi apparatus. In this first hypothesis, those enzymes may stay in the first saccules that are motionless by definition. In the second hypothesis, namely the saccule maturation (see Fig. 2(b)), saccules are still disconnected but follow an anterograde movement which supports the transport of proteins. Here, vesicles move along a retrograde flow in order to return enzymes that function early in the pathway to the cis region. It must be said that while the two previous hypotheses are well anchored in the biological knowledge, none of them take the perforation that appears in some pictures into account. The third hypothesis, which promotes a continuous Golgi apparatus, takes into consideration the saccules perforation. The representation introduced in Fig. 2(c) results from discussions we had with biolologists and give a first topologyoriented insight of this new hypothesis. This continuous hypothesis does not rely on any vesicle transportation. On the contrary, it considers a continuous membranes flow (see Fig. 2(c)) emerging from the endoplasmic reticulum. Indeed, observed endoplasmic reticulum fragments and vesicles are interpreted in this hypothesis as small sections of a tubular network that connects the saccules $(\mathbf{T})$. In this case, proteins may follow the membrane flow and diffuse from one saccule to another along the tubes while enzymes may diffuse following a retrograde movement. Moreover, in this last hypothesis, the saccules perforation may explain the creation of the secretory granules by the rupture of the junctions resulting from the perforation.

It seems clear that among the numerous features involved in the Golgi apparatus (from the precise shape of the object to the different molecule flows), the role played by the topology is decisive. Thus, a relevant abstraction of the previous Golgi Apparatus hypotheses must handle this component. In [Poudret et al., 2007], we proposed a topology-based abstraction illustrated on a simple biological process inspired by the gap junction phenomenon [Alberts, 2002]. Here, we want to show that our framework is well-adapted to the modelling of complex biological systems where, like in the Golgi apparatus, the neighbouring relations that exist between compartments are relevant features.

\section{Topology-based geometric modelling for biological cellular processes}

\subsection{Topology-based geometric modelling}

In order to take into account the biological compartments into our model, we rely on the topology-based geometric modelling (topological modelling for short). This field of the computer graphics deals with the representation of the objects structure (their decomposition into topological units: vertices, edges, faces and volumes) and of the 


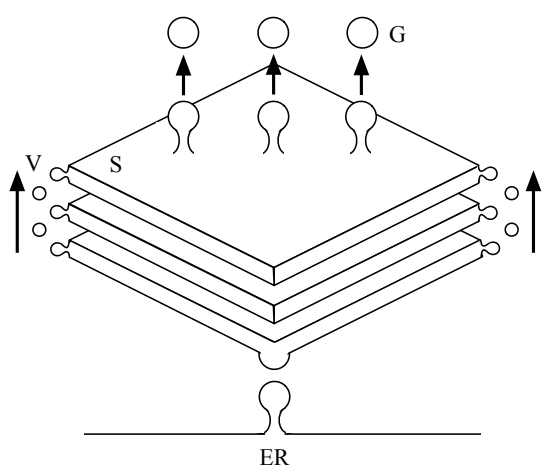

(a) Vesicular excretion

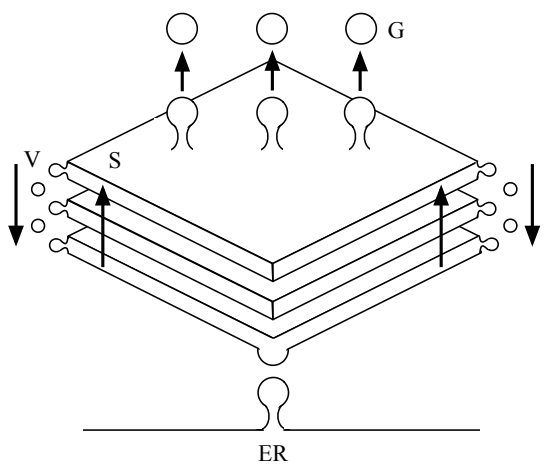

(b) Saccule maturation

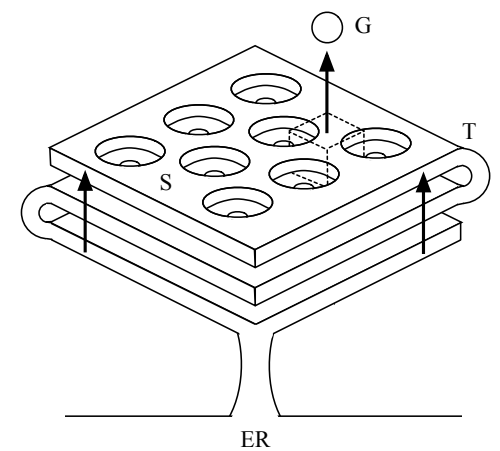

(c) Continuous membrane flow

Fig. 2 Three hypotheses on the Golgi apparatus

neighbouring relations that exist between topological units. Among numerous topological models, we choose the $n$-dimensional generalised map [Lienhardt, 1989] ( $n$-G-maps for short). It defines the topology of an $n$-dimensional space subdivision and allows the representation of a large class of objects ${ }^{1}$. This topological model has the advantage of providing a homogeneous mathematical definition for all dimensions. This genericity allows one to easily develop robust softwares.

On Fig. 3, a 2-dimensional object (see Fig. 3(a)) is successively decomposed into topological units: faces (see Fig. 3(b)), edges (see Fig. 3(c)) and vertices (see Fig. 3(d)). These vertices, also called darts, are the basic elements of the $n$-G-maps. Labelled graph edges are used in order to recover the neighbouring relations (see Fig. 3(e)). The label of an edge depends on the nature of the neighbouring relation that is symbolised by the edge. For instance, the $\alpha_{2}$-edge between $v$ and $v^{\prime}$ illustrates the sticking of the two faces (2-dimensional topological units) that include $v$ and $v^{\prime}$ on the original picture (see faces $f$ and $f^{\prime}$ on Fig. 3(a)). Here is the mathematical definition of an $n$-G-map:

Definition 1 ( $n$-G-map) Let $n \geq 0$. An $n$-G-map is an edge-labelled graph $G=$ $\left(V_{G}, E_{G}\right)$ with labels in $\Sigma_{E}=\left\{\alpha_{0}, \ldots, \alpha_{n}\right\}$, such that:

- for all $v \in V_{G}, l \in \Sigma_{E}$, there exists a unique $v^{\prime} \in V$ such that $\left(v, l, v^{\prime}\right) \in E_{G}$;

\footnotetext{
1 Quasi-manifolds, orientable or not.
} 


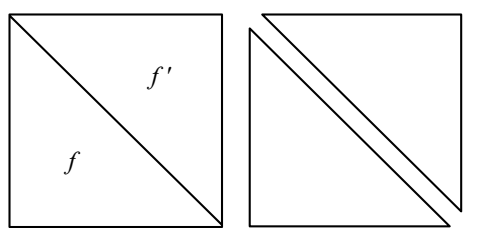

(b)

(a)

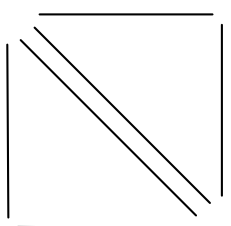

(c)

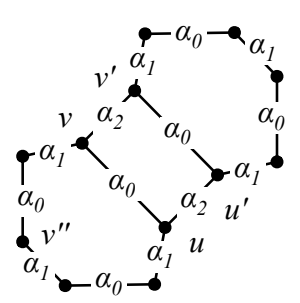

(e)

Fig. 3 2-G-map intuition

- for each $v \in V_{G}$, for all $\alpha_{i}, \alpha_{j} \in \Sigma_{E}$ such that $0 \leq i<i+2 \leq j \leq n$, there exists a cycle $\left(\alpha_{i}, \alpha_{j}, \alpha_{i}, \alpha_{j}\right)$ that reaches $v$.

On the border of the objects, some darts do not have all possible neighbours. For instance, on Fig. 3(e) the dart $v^{\prime \prime}$ is not linked to another dart by an $\alpha_{2}$-edge. However, according to the first point of definition all darts must have one incident label for each dimension. Thus, if a dart is not linked to another dart by an $\alpha_{i}$-edge $(0 \leq i \leq n)$, it exists an implicit $\alpha_{i}$-loop that links the dart to itself. For instance, on Fig. 3(e), there is an implicit $\alpha_{2}$-loop incident to vertex $v^{\prime \prime}$. The second point of the definition means that if two $i$-dimensional units are stuck, they must be stuck along a $(i-1)$ dimensional unit (this is a quasi-manifold condition). For instance, on Fig. 3(a), the faces (2-dimensional units) $f$ and $f^{\prime}$ are stuck along an edge (1-dimensional unit). In the corresponding 2-G-map, this property is translated into the presence of a cycle $\left(\alpha_{0}, \alpha_{2}, \alpha_{0}, \alpha_{2}\right)$ that reaches $v$ on Fig. 3(e). Thus, since darts $v$ and $v^{\prime}$ are linked with an $\alpha_{2}$-edge, then darts $u$ and $u^{\prime}$ are linked with an $\alpha_{2}$-edge as well.

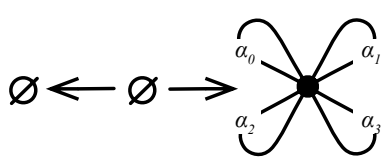

(a) addition rule

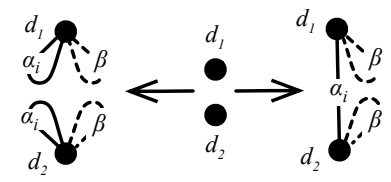

(b) $i$-sew meta-rule

Fig. 4 rules and meta-rules 
In order to edit topological objects, computer scientists have defined many topological operations on the $n$-G-maps. Moreover, it has been established that all of these operations can be decomposed into four basic operations: dart addition, dart suppression, dart $i$-sew and dart $i$-unsew (these two last operations consist in, respectively, sticking and unsticking two $i$-dimensional topological units along two isomorphic ${ }^{2}(i-1)$ dimensional topological units).

In [Poudret et al., 2007], we have formally expressed the basic operations as graph transformation rules [Ehrig et al., 2006]. Classical rules are sufficient to express the dart addition (see Fig. 4(a)) and dart suppression (this new rule is obtained by swapping the left-hand and right-hand sides of the dart addition rule). Nevertheless, they are not sufficient to represent the dart $i$-sew and $i$-unsew operations. Indeed, the graph transformation to perform for sticking (resp. unsticking/separating) two topological units together depends on the size of these units. For instance, sticking two triangular faces together implies adding $6 \alpha_{2}$-edges while sticking two square faces together implies adding $8 \alpha_{2}$-edges. As it is clearly unreasonable to introduce as many rules as all the potential sizes of the topological units, we introduce the notion of graph transformation meta-rule that abstracts this infinite set of classical rules.

Definition 2 (meta-rule definition) Let us consider $\Sigma_{E}$ a set of labels and $\beta \notin \Sigma_{E}$ a new label. A graph transformation meta-rule on $\beta$, noted $L \leftarrow K \rightarrow R$, is a graph transformation rule where $L, K$ and $R$ are edge-labelled graphs with labels in $\Sigma_{E} \cup\{\beta\}$ and satisfying both following properties:

- for each edge in $L$ (resp. $R$ ) of the form $\left(v, \beta, v^{\prime}\right)$, then $v=v^{\prime}$;

- there exists at least in $L$ an edge of the form $(v, \beta, v)$. Graphically, $\beta$-edges are noted with dotted lines.

In [Poudret et al., 2007], we define the translation of such a meta-rule into a set of classical rules. The dart $i$-sew meta-rule is introduced in Fig. 4(b) (the $i$-unsew metarule is obtained by simply swapping the left-hand and right-hand side of the $i$-sew meta-rule). On this figure, the $\beta$-edges represent the topological units that parameterise the meta-rule. In short, the $i$-sew meta-rule may be understood as follows. It matches two isomorphic $i$-dimensional topological units (abstracted with $\beta$-edges) such that all $\alpha_{i}$-edges are loops. Then, it links the vertices of both units with an $\alpha_{i}$-edge.

\subsection{Embedding}

In order to model biological cellular processes we may want to attribute different kinds of information to the topological units, that is to say to embed them. For instance, we may want to attach types, biochemical data, geometric data (when the biological observation allows it), etc. to the volumes that abstract the biological compartments. Thus, we may want to write transformation rules whose application depends on embedding and that modify embedding information. In [Poudret et al., 2007], we introduced a language in order to write embedding expressions. Here is an example of expressions (here, we give a simplified syntax whose meaning can be easily understood from the usual convention) which can be related to the 3-sew meta-rule (see Fig. 4(b), with

\footnotetext{
2 Roughly speaking, two topological structures are said to be isomorphic if they are superposable.
} 


$$
\begin{aligned}
& i=3) \text { : } \\
& \left(3 \_ \text {distance }\left(d_{1}, d_{2}\right) \leq \epsilon\right) \wedge\left(3 \_t y p e\left(d_{1}\right)=\text { Vesicle }\right) \wedge\left(3 \_t y p e\left(d_{2}\right)=\text { Saccule }\right) \\
& \text { 3_update_position }\left(d_{1}\right)
\end{aligned}
$$

Pre-condition (1) is used to restrict the application of the rule. The prefix 3 of 3_distance means that we consider the distance between 3-dimensional topological units (i.e. volumes). Thus, this condition means that the volumes which contain the darts $d_{1}$ and $d_{2}$ may be glued only if they are sufficiently close to each other (according to a small distance, denoted $\epsilon$ here) and if the volume that contains $d_{1}$ (respectively $d_{2}$ ) is of type $V$ esicle (respectively Saccule). Finally, the post-condition (2) updates the position of the volumes that abstract the vesicles (to geometrically stick it on the saccules).

\section{Topology-based abstraction of Golgi Apparatus hypotheses}

\subsection{Topological models of the Golgi Apparatus}

In Section 1.2, we introduced three hypotheses that may explain the behavior of the Golgi apparatus. Two of these hypotheses are well-known and implicate vesicles in the transport of proteins while the third hypothesis is more recent and involves a continuous membrane flow in a tubular network. In this section, we use our topology-based approach to model on one hand one of the vesicle hypotheses and on the other hand the continuous membrane flow hypothesis. In the case of the vesicle hypotheses, we focus on the saccule maturation hypothesis, which involves several different phenomena (both saccule and vesicle movements) and is therefore more subtle than the hypothesis that simply involves excretion of vesicles. Moreover, the two vesicular hypotheses are strictly identical from the topological point of view (only the dynamics differ) while the continuous membrane flow introduces significant topological differences (connected and perforated saccules).

Fig. 5 illustrates 3-G-map topological representations of both hypotheses. The plate stack model (see Fig. 5(a)) represents the saccule maturation hypothesis and the tower model (see Fig. 5(b)) represents the continuous membrane flow hypothesis.

The topology-based geometric modelling allows one to easily abstract geometry and to focus on pure topology which is, as we said, the most relevant distinction between studied Golgi apparatus hypotheses. When it is necessary, geometric shapes can be embedded on the topological units. On Fig. 5, the geometry is basic (here, the object are said to be polyhedric) but the topological differences between hypotheses are captured. The first distinction is the connection between the saccules $(\mathbf{S})$. The proteins are transported through vesicles $(\mathbf{V})$ in the plate stack while they diffuse into tubes $(\mathbf{T})$ that connect the saccules in the tower model. As we see, we choose to abstract both saccules and vesicles with volumes on which we embed concentrations that abstract proteins (concentration gradients are modelled by subdividing volumes and associating different concentrations on each subdivision). Moreover, from a topological point of view, a tube between two saccules is represented with a volume stuck between the connected saccules. Because topological models allow one to handle border of volumes, we abstract the transport of proteins associating permeability on faces that connect saccules to tubes. The second topological distinction concerns the creation of the secretory granules. In the plate stack, the secretory granules $(\mathbf{G})$ bud out from the trans 


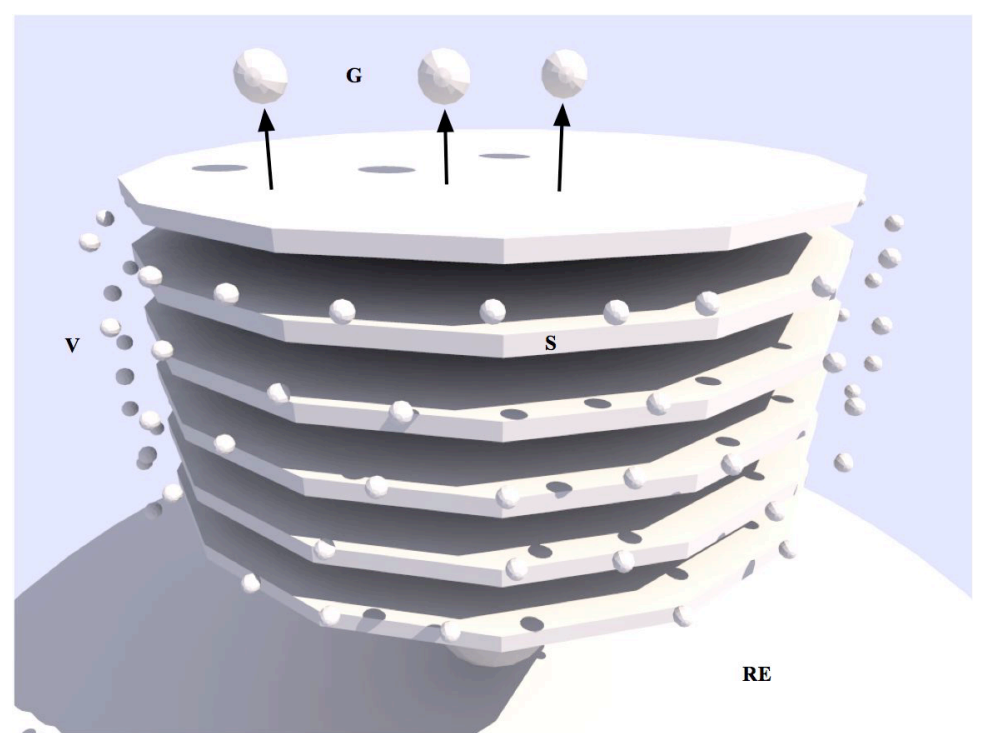

(a) Plate stack model

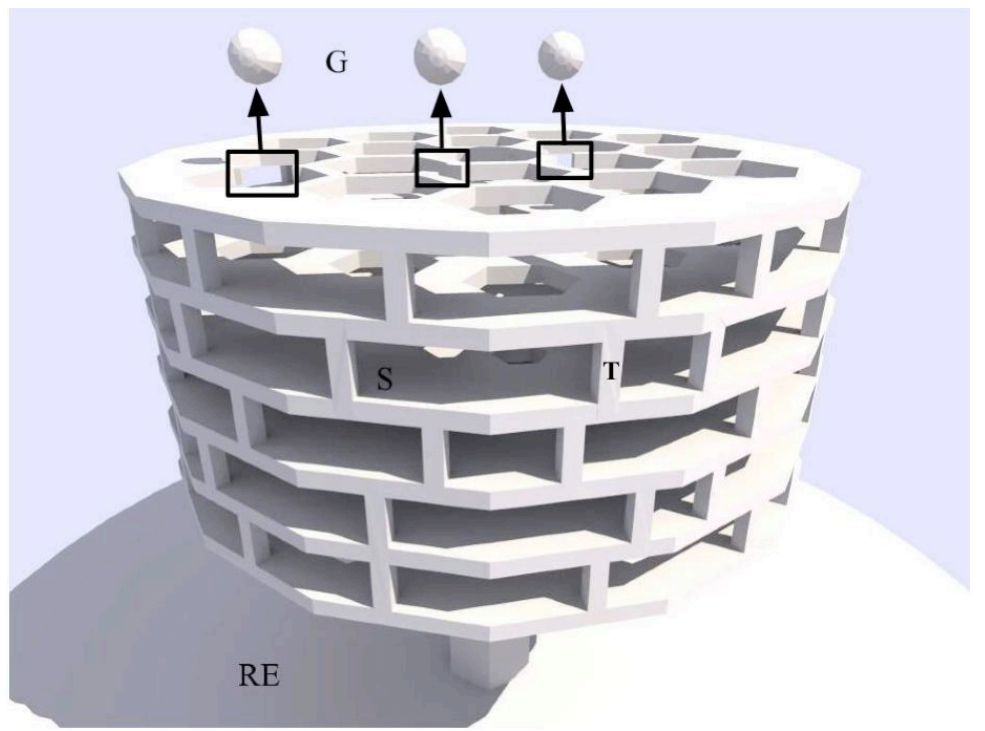

(b) Tower model

Fig. 5 3-G-map topological representation of Golgi Apparatus

face (see arrows of Fig. 5(a)) while in the tower model, they are constituted of saccule pieces that result from the rupture (framed on Fig. 5(b)) of the bee nest structure that abstracts the perforation (according to the biologists, the perforation appears progressively from the cis face to the trans face). Finally, small parts of the endoplasmic reticulum aggregate into saccules in the first model, while the endoplasmic reticulum is connected to the cis face in the second one. 


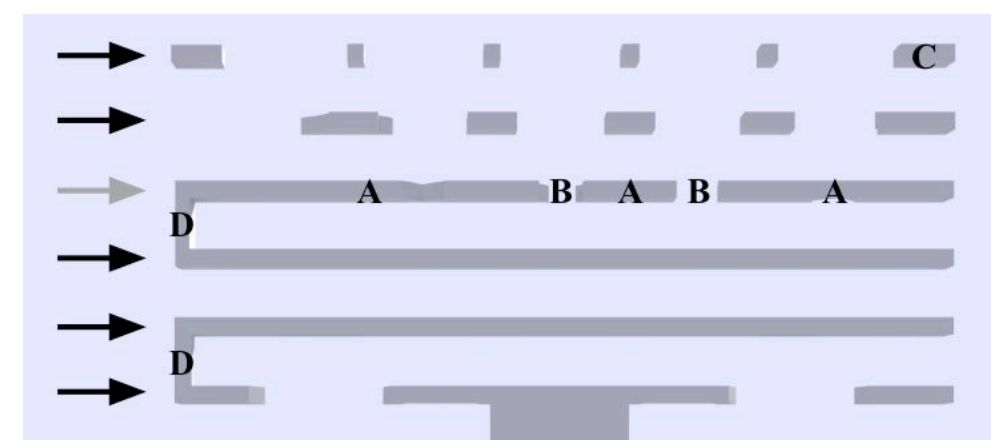

Fig. 6 Vertical section of the tower model

Both models have been elaborated by following a loop of topological model refinements. Biologists have deeply analysed intermediate models by proposing many topological updates. This is particularly true for the continuous membrane flow for which the tower model presented in Fig. 5(b) gives a first insight of its precise topological structure. The vertical section of tower model presented in Fig. 6 has played an important role in the biologists validation process. On this figure, we observe sections of the six saccules (indicated with arrows) of Fig. 5(b) and we notice that some sections show irregularities. For instance, the section indicated with the grey arrow presents alternately plain regions (A) and empty regions (B). According to the biologists, this observation is certainly correlated to the light and dark regions that appears on the saccule sections of electron micrographs (see Fig. 1(a)). Moreover, we see that a dark region on the border of the saccule stack $(\mathbf{C})$ may be easily confused with the vesicles that surround the stack (see V on Fig. 1(a)). Finally, like in some electron micrographs, the tower section presents some bridges (D) that connect the saccule section (here, they are remains of the tubular network). These remarks show that our tower model, which has been initiated by the biologist observation of perforations (see Fig. 1(b)) and by the interpretation of vesicles as small sections of tubes, is fully compatible with the electron micrographes that are usually used to promote the classical vesicular hypotheses. Let us point out that the plate stack model, that abstracts one of the classical Golgi apparatus hypotheses, cannot really explain (by construction) the irregularities (light regions, perforations and connections between saccules) that appear one electron micrographes. Thus, the tower model is the first contribution of our topological abstraction process since it has been shown to be consistent with the biological observations.

\subsection{Animating the topological models}

In Section 2, we have introduced a means to write rules that transform topological objects. In order to animate the plate stack and the tower model, we have to write the rules that capture their dynamics. In this section, we give two examples of such rules. They are introduced in Fig. 7 and Fig. 8.

The first rule is dedicated to the plate stack model. It models the gluing of a vesicle with a saccule which initiates their fusion. Fig. 7(b) introduces a simplified representation of the matched pattern, it contains a vesicle $(\mathbf{V})$ close to a saccule 


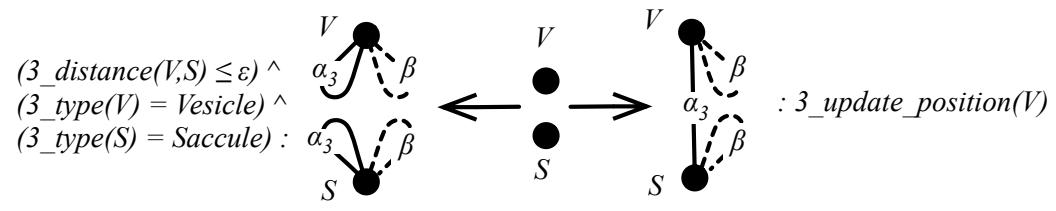

(a) Transformation rule

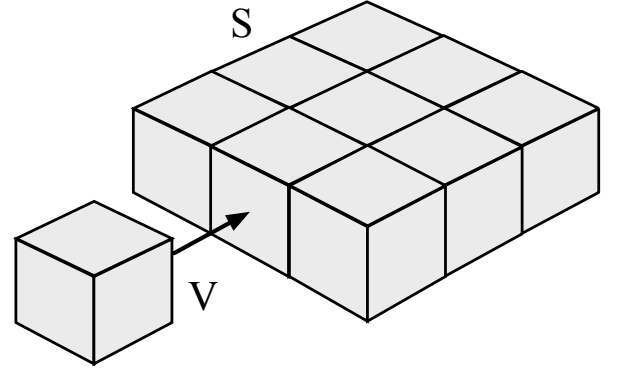

(b) Matched pattern

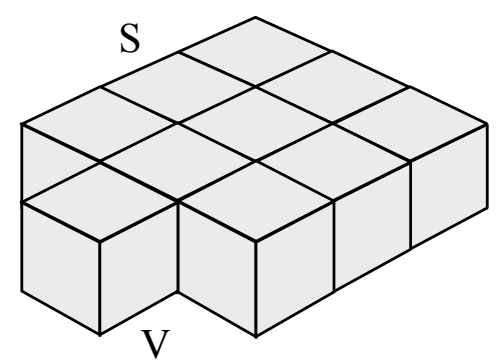

(c) Result

Fig. 7 Gluing a vesicle with a saccule

(S). The transformation rule (see Fig. 7(a)) glues them (using a 3-sew operation) and updates the position of the glued vesicle (see Fig. 7(c)). On the rule, $V$ and $S$ are respectively darts of vesicle and saccule, and $\beta$-edges match volumes.

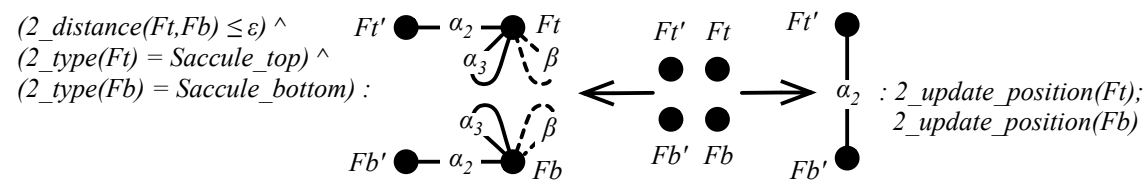

(a) Transformation rule

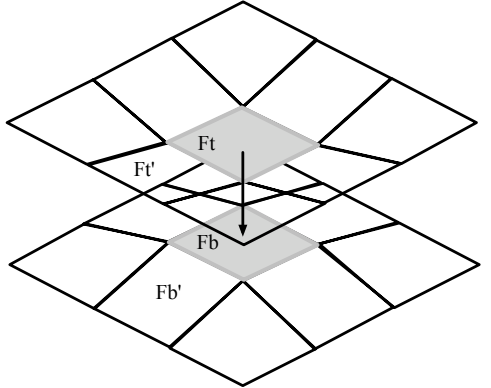

(b) Matched pattern

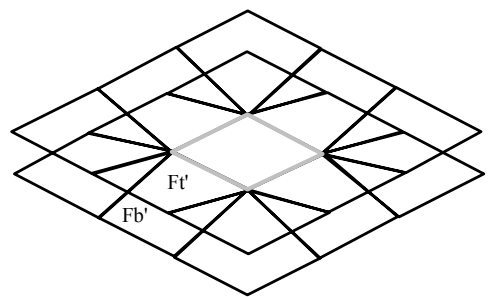

(c) Result

Fig. 8 Perforating a saccule

The second rule is dedicated to the tower model. It provides a means to perforate the saccules (which is one of the behavior at the root of the continuous membrane flow 
hypothesis). The matched pattern (see Fig. 8(b)) contains two close faces (Ft) and $(\mathbf{F b})$ that belong to the same saccule (one is on the top, the other on the bottom). The rule (see Fig. 8(a)) executes the perforation removing faces $(\mathbf{F t})$ and $(\mathbf{F b})$ and linking their neighbours (see Fig. 8(c)). On the rule, $F t$ and $F b$ are respectively darts of removed top and bottom faces, $F t^{\prime}$ and $F b^{\prime}$ are neighbour faces, and $\beta$ matches faces.

These two examples are well-representative of the transformation rules we need in order to animate the plate stack and tower models. For instance, in the plate stack model, most of the topological operations consist in sticking (it is the case of the example rule) or unsticking topological objects. The stick operation is used to aggregate the pieces of endoplasmic reticulum that constitute a new saccule while the unstick operation is used to abstract the budding out of vesicles and secretory granules. In these rules, only the embedding pre-condition and post-condition differ.

We should notice that geometry plays a decisive role in animation processes. The geometric data that influence the biological function we are abstracting are handled in the embedding condition associated to the rules. For instance, when a rule is applied it can take the proximity of objects into account. Other phenomena, e.g. collision dectection between vesicles or secretory granules, are ignored. In fact, many of them only influence the visual rendering, and we do not consider this issue as significant in our context.

\section{Ongoing work: toward a topological discrimination of Golgi Apparatus hypotheses}

In section 3 we have used our framework in order to introduce a topology-based abstraction of two Golgi apparatus hypotheses: the saccule maturation and the continuous membrane flow. For both of them, we have defined a topological model (respectively the plate stack model and the tower model) and have given examples of rules that handle their dynamics. Thus, we have shown that our framework is well adapted to the modelling of biological processes that, like the Golgi apparatus, involve strong topological features.

Nevertheless, the definition of graph transformation meta-rules is mandatory for animating the topological models but is not sufficient to simulate such complex systems. The rules only define the syntactic part of the simulations, in other words, they define what kind of transformations the simulator performs. Our ongoing work consists in exploring what kind of strategies have to be taken into account when applying transformation rules in order to play simulations. In order to help the biologists to better understand a given biological complex system, we furthermore aim at introducing a computer-aided methodology for analysing topology and dynamics of different hypotheses associated to the biological system. Our goal is not to build an accurate model, but instead, we would rather discriminate between the different models and choose the one which best approximates the observed phenomena. However, to properly define our discrimination methodology, we need at first to define the parameters of the models. 
Table 1 Simulation parameters

\begin{tabular}{llll}
\hline Parameters & Value & Plate stack model & Tower model \\
\hline Membrane tickness & $7 \eta m$ & Yes & Yes \\
Number of saccules & 6 & Yes & Yes \\
Saccule thickness & $30 \eta m$ & Yes & Yes \\
Saccule length & $50 \times 30 \eta m$ & Yes & Yes \\
Secretory granule diameter & $120 \eta m$ & Yes & Yes \\
Number of proteins in a granule & 600 & Yes & Yes \\
\hline Vesicle diameter & $60 \eta m$ & Yes & No \\
Tube diameter & $60 \eta m$ & No & Yes \\
\hline Membrane quantity & OUT & Yes & Yes \\
ATP consumption & OUT & Yes & Yes \\
\hline
\end{tabular}

\subsection{Parameters}

Table 1 gives example of parameters that have been discussed with biologists about discrimination of Golgi apparatus hypotheses. The first column displays the name of the parameters. An approximation of their value when they exist (according to the biological state of the art) appears in the second column. A value OUT means that the parameter is computed within the simulation. The third and fourth columns tell whether a given parameter makes sense in respectively, the plate stack model and the tower model. The given set of parameters is not exhaustive but contains the parameters considered by the biologists as the most relevant for the comparison of the two models.

The first six parameters are input parameters (their values are given by the biologists, according to the observations) and are used to initialize the topological models. They are embedded on the topological units at the beginning of the simulation and can be refined as we discuss in the next section. The vesicle and tube diameter are also input parameters but fit to only one topological model (respectively the plate stack model and the tower model). Let us remember that depending on the hypothesis we are considering (vesicular or continuous membrane flow hypothesis), the same pieces of an electron micrograph can be considered as vesicle or tube section. Thus, vesicle and tube diameter must be the same. In other words, updating one of them implies to update the other one. Finally, the last two parameters: membrane quantity and ATP (it is the energetic unit of the cell) consumption have been chosen among others to discriminate the topological models. Biologists think that the quantity of membrane within the Golgi apparatus (vesicles and secretory granules take part of it) must be constant in time. Thus, if reaching a wanted quantity of transported proteins within the Golgi implies to break this property in one model, this could allow the discrimination of the two models. In the same manner, the fact that the quantity of consumed ATP reaches a critical level could be discriminating too.

\subsection{Discrimination methodology}

The proposed methodology, which is based on successive simulations of the topological models that implement the hypotheses, is illustrated on Fig. 9. The figure only takes into account two topological models but can easily be extended. The different kinds of parameters described in the previous sections are introduced. The input parameters 


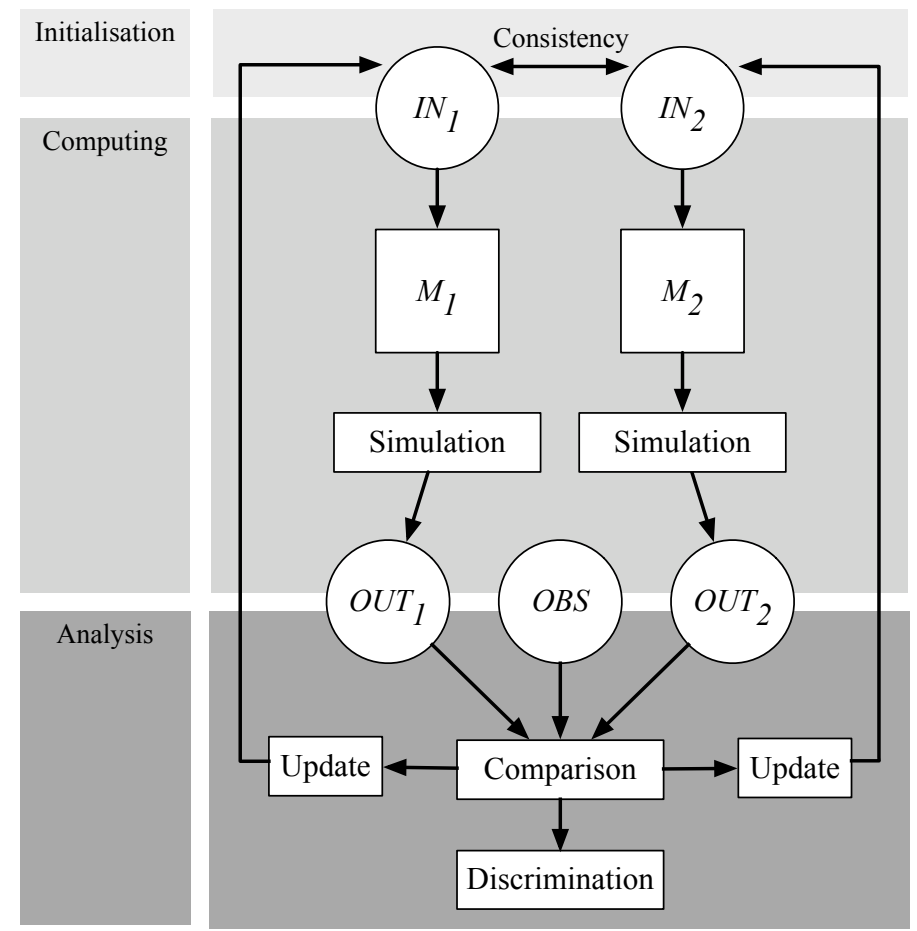

Fig. 9 Models discrimination loop

$\left(I N_{1}\right.$ and $\left.I N_{2}\right)$ are used to initialize the simulations of, respectively, topological models $M_{1}$ and $M_{2}$ that implement the selected hypotheses. Note that, as discussed earlier, some parameters can be specific to only one model, but are correlated to parameters of the other model. This consistency between parameters of $M_{1}$ and $M_{2}$ is necessary for the models discriminating process. $O U T_{1}$ and $O U T_{2}$ parameters result from the simulations of, respectively, $M_{1}$ and $M_{2}$ (for instance, the flow of excreted proteins are output parameters for both models). The results of the simulations are compared with biological experimental observations ( $O B S$ on the figure). Our methodology then consists in a refinement process, that is modifying the set of input parameters according to the observations. Note that the models must not be refined independently: the updates still guarantee the consistency between $I N_{1}$ and $I N_{2}$. Thereafter, we reiterate the simulation, comparison and refinement processes. This loop aims at making both models converge toward the experimental observations. We then select the model that better approximates the observation or eliminate the one that do not converge toward the desired output values. More precisely, a model is given up when one can no longer update parameters in a satisfactory manner. If such a model is not consistent with available biological data, it is refuted and only the other model is considered for further analysis. Our methodology is intentionally simplistic. Our goal is to consider models as simple and abstract as possible to be analysed by biologist experts. When necessary, according to observation or in order to discriminate models, detailed elements are gradually incorporated in the models under discrimination in order to make models more complex, up until the moment biologist experts can discard one of the models. 
Thus, there is a trade-off between abstraction of the models and the need of information to discriminate models. The development of finer models becomes useless even if these finer models would be more plausible with respect to the real biological processes under consideration.

\section{Conclusion}

In this paper we develop a method of modelling cellular processes that takes topology into account. This method facilitates a better understanding of the dynamics of those cellular processes that strongly depend on compartmentalization. It relies on topologybased geometric modelling in order to represent compartments with their neighbouring relations and on transformation rules that allow simultaneous simulation of topological, geometric and biochemical mechanisms. This topology-based approach is applied to the Golgi apparatus, a complex biological system where topology places a key (but poorly understood) role. We first study two very different topological representations based on two of the three principal hypotheses about the topology of the Golgi: in the plate stack model, saccules are disconnected and proteins move from one saccule to another via vesicles, while in the tower model (which is based on a new hypothesis about the dynamics of the Golgi), saccules are connected with tubes that allow proteins to cross the Golgi. A 2-dimensional section shows that only the tower model is compatible with the available biological observations. Finally, these topological models can be animated using transformation rules that are determined by the geometrical and biochemical data and that determine both these data and the topology itself.

\section{Acknowledgments}

The authors thank Jean-Marc Verbavatz, Janine Guespin and Alain Rambourg, members of GolgiTop working group initiated by the Epigenomics Project (Génopole, France), for regularly sharing their deep knowledge of the Golgi apparatus hypotheses. We thank Vic Norris for help with English. Finally, we thank Pierre-François Léon for help with rendering the plate stack and tower model pictures.

\section{References}

[Alberts, 2002] Alberts, B. (2002). Molecular biology of the cell, 4th ed. Garland Science.

[Bernot et al., 2004] Bernot, G., Comet, J.-P., Richard, A., and Guespin, J. (2004). Application of formal methods to biological regulatory networks: Extending Thomas' asynchronous logical approach with temporal logic. Journal of Theoretical Biology, 229(3):339-347.

[Calzone et al., 2006] Calzone, L., Fages, F., and Soliman, S. (2006). Biocham: an environment for modeling biological systems and formalizing experimental knowledge. Bioinformatics, 22(14):1805-1807.

[Cardelli, 2005] Cardelli, L. (2005). Brane calculi. interactions of biological membranes. In Proc. Computational Methods in Systems Biology, volume 3082 of Lecture Notes in BioInformatics, pages 257-280. Springer.

[Ehrig et al., 2006] Ehrig, H., Ehrig, K., Prange, U., and Taentzer, G. (2006). Fundamentals of Algebraic Graph Transformation (Monographs in Theoretical Computer Science. An EATCS Series). Springer, Secaucus, NJ, USA.

[Eungdamrong and Iyengar, 2007] Eungdamrong, N. and Iyengar, R. (2007). Compartmentspecific feedback loop and regulated trafficking can result in sustained activation of ras at the Golgi. Biophys. J., 92(3):808-815. 
[Képès et al., 2004] Képès, F., Rambourg, A., and Satiat-Jeunemaître, B. (2004). Morphodynamics of the secretory pathway. International review of cytology, 242:55-120.

[Lienhardt, 1989] Lienhardt, P. (1989). Subdivision of n-dimensional spaces and n-dimensional generalized maps. In $S C G^{\prime} 89$, pages 228-236. ACM Press.

[Mateus et al., 2007] Mateus, D., Gallois, J.-P., Comet, J.-P., and Le Gall, P. (2007). Symbolic modeling of genetic regulatory networks. Journal of Bioinformatics and Computational Biology.

[Poudret et al., 2007] Poudret, M., Comet, J.-P., Le Gall, P., Arnould, A., and Meseure, P. (2007). Topology-based geometric modelling for biological cellular processes. In LATA'0\%. Available at http://grammars.grlmc.com/LATA2007/proc.html.

[Presley et al., 2002] Presley, J. F., Ward, T. H., Pfeifer, A. C., Siggia, E. D., Phair, R. D., and Lippincott-Schwartz, J. (2002). Dissection of COPI and Arf1 dynamics in vivo and role in Golgi membrane transport. Nature, 417:187-193.

[Rambourg et al., 1979] Rambourg, A., Clermont, Y., and L., H. (1979). Three-dimensional architecture of the golgi apparatus in sertoli cells of the rat. American Journal of Anatomy, 154(4):455-476.

[Regev et al., 2004] Regev, A., Panina, E. M., Silverman, W., Cardelli, L., and Shapiro, E. (2004). Bioambients: an abstraction for biological compartments. Theor. Comput. Sci., 325(1):141-167. 